\title{
Digital Twin-Based Modeling of Complex Systems for Smart Aging
}

\author{
Yiyi Deng $\mathbb{1}$ \\ College of Finance and Statistic, North Campus, Hunan University, Changsha City 410079, Hunan Province, China \\ Correspondence should be addressed to Yiyi Deng; yiyi_deng@hnu.edu.cn
}

Received 12 December 2021; Revised 4 January 2022; Accepted 7 January 2022; Published 27 January 2022

Academic Editor: Gengxin Sun

Copyright (c) 2022 Yiyi Deng. This is an open access article distributed under the Creative Commons Attribution License, which permits unrestricted use, distribution, and reproduction in any medium, provided the original work is properly cited.

In this paper, we use digital twin technology to conduct in-depth analysis and research on the modeling of complex systems for smart aging. The solution of the digital twin model based on a big data platform is proposed, and the problem of asynchronous and incomplete digital twin real-time monitoring data is solved, and the algorithm is applied to the digital twin model based on the big data platform for data preprocessing to achieve better results. To improve the real-time data transmission, the OPCUA information modeling method is optimized by using node merging and adaptive compression, which statutes the system data and achieves the effect of information fusion. The Web protocol is also used to unify the digital twin information interaction form. After experimental testing, the effectiveness of the information modeling scheme designed in this paper is verified. The fall detection results based on the digital twin selected thresholds are significantly better than the experimental results of the manually set threshold method; the thresholds set by the digital twin can more accurately identify daily behaviors, especially the more violent daily behaviors such as lying down, and the accurate alarm rate of the falling behavior reaches $92.5 \%$. In contrast, the artificially set thresholds have a lower overall recognition rate for human behaviors and are prone to misclassification, with a misclassification rate of 3.8\%. Therefore, it can be determined that using the digital twin method to set feature thresholds is better than the manual setting threshold method in terms of detection accuracy, and the digital twin method is chosen to select the thresholds for each stage of the fall process in this project. The validation results demonstrate the system's excellence in information interaction, optimization of numerical analysis, and display of results for smart aging.

\section{Introduction}

Population aging and information technology are two important trends in the development of modern society, and the increasing demand for elderly care services poses a challenge to the unbalanced and insufficient development of China's elderly care service system at present [1]. Embedding intelligent senior care supported by information technology and intelligence into the senior care service system is not only an objective need to actively cope with the aging population but also an opportunity of the times to meet the development trend of the information society and seize the opportunity to promote the level of social governance [2]. In the field of elderly care, the contradiction between the growing pursuit of a better life for the elderly in their old age and the unbalanced and insufficient development of the elderly care industry is prominent. The traditional elderly service model mainly includes family elderly care, community elderly care, and institutional elderly care. With the "4-2-2" family structure and large-scale aging, it is difficult for the traditional elderly care model to adapt to the needs of the elderly in the new era. To promote the development of the senior care industry, the "two legs" of stimulating consumption and deepening the supply-side structural reform must be launched at the same time. Every breakthrough in science and technology will promote great changes in economic society and human society [3]. Traditional elderly care services can only be provided through manual services, offline. With the continuous improvement of technology, we have applied modern information technology, intelligent products, and services to elderly care services, which can be realized through a combination of online and offline services. In addition to this, the elderly is taking the initiative to adapt to the new situation, changing their concept of elderly care and readily accepting the convenience brought by technology. 
Digital twin (DT) is also translated as digital twins, digital mirror, or digital mapping. It is a simulation process that makes full use of physical models, sensors, operational history, and other data to integrate multidisciplinary, multiphysical quantities and multiple scales, reflecting the whole life cycle process of the corresponding physical equipment and products by mapping the realization to the physical space in the digital space pair [4]. The digital twin system is essentially a functional system that combines physical entities and twin models into a continuous process control optimization, so the digital twin has also become one of the core technologies of Cyber-Physical Systems (CPS). Gartner, a leading IT industry consulting and research firm, has listed digital twin as one of the top 10 strategic technology trends of the future for three consecutive years from 2017 to 2019 and considers digital twin to include the following: metadata, situation, event data, and analytics, a multifaceted software model of a thing or system that relies on sensors as well as other data to understand its situation, respond to change, improve operations, and add value [5].

Looking globally, population aging has become a global trend and is no longer confined to a single region or country. In 2017, the number of people aged 60 and above reached 962 million worldwide, and the number of people in that age group is expected to reach 2.1 billion by 2050. The proportion of older people in the total population rose from $8 \%$ in 1950 to $10 \%$ in 2000 , and, at the rate of $2 \%$ annual growth of the older population, the proportion of older people will reach $21 \%$ by 2050 . Based on the above background trend of population aging and the problems of traditional senior care services, smart senior care begins to emerge [6]. In this paper, the content and characteristics of the elderly service are combined with the innovative way and content of the elderly service. That is, taking the community as the carrier, while embedding functions, resources, and interactions, it embeds intelligent technical means based on the Internet and the Internet of Things to meet the physical and psychological multifaceted needs of the elderly, dispose of social risks, and improve the quality of elderly services.

\section{Related Works}

NASA (National Aeronautics and Space Administration) first introduced the concept of "twins" in its Apollo program. In 2003, Professor Michael Grieves introduced the concept of "a digital representation of physical products," which combines the physical space, digital space, and both in a digital twin. In 2011, the U.S. Air Force Laboratory developed the concept of the digital twin to address future vehicle maintenance and lifetime prediction in complex environments and led to its further development [7]. A digital twin of an aircraft airframe as a hyperrealistic model of the airframe was built and maintained for digital diagnosis and analysis of the real airframe. At this point, the digital twin initially moved from the conceptual model stage to the practice and application stage, and its nature and connotations were described and studied in greater depth, highlighting the hierarchical, integrated, hyperrealistic, extensive, consistent, and computable nature of the digital twin [8].
Some developed countries in the West have already entered the aging society as early as the 1980s, and, due to their early start, they have gradually formed a more comprehensive intelligent elderly care service system, the achievements of which are mainly focused on remote medical monitoring, intelligent elderly robots, and wearable assistive devices for the elderly [9]. Honor in the United States has developed an online marketplace platform for elderly care services. Caregivers need to fill in their qualifications, skills, service types, service hours, and other relevant information on the platform when they first log in, while the elderly also need to improve their personal information and service needs, and then the Honor platform will match the information of caregivers with the needs of the elderly according to the supply and demand relationship to achieve service. The Etchemendy research group in Spain has also developed a health management platform for the elderly, which differs from Honor in that the Etchemendy research group pays more attention to the emotional changes of the elderly while paying attention to the health data of the elderly [10]. Predictive model is an important part of predictive control. Its main function is to predict the future state or output of the controlled object based on its historical information and future input, especially for nonlinear predictive control systems. Predictive control does not have strict mandatory requirements for the structure of the predictive model, but its control effect largely depends on the performance of the predictive model. Tass, a Swedish company, has developed an Android-based mobile health monitoring system, which uses various sensors to collect data on human body signs (e.g., heart rate, blood pressure, blood sugar, etc.) and displays these data on the mobile terminal in real time, while the system can also provide the elderly with targeted health advice based on the analysis results of the sign data. Carnegie Mellon University has developed the Narrator System, which uses artificial intelligence technology to statistically analyze the behavioral activities of the elderly and generate activity reports for family members and doctors to view, so that family members and doctors can have a better understanding of the elderly's activity status and prevent accidents [11].

Although the research of intelligent elderly systems in China started late compared with foreign countries, in the past 20 years, various experts and scholars, government, and social organizations have successively researched and achieved certain results on the intelligent elderly system [12]. Li Bin designed a new portable ECG monitor based on ARM Cortex-M3, which not only satisfies real-time QRS wave detection but also realizes the functions of human-computer interaction, waveform playback, arrhythmia analysis, and disease alarm; Zhou Ziyi built a health management system for the elderly in nursing institutions, which analyzes the characteristics of the elderly health data and adopts data mining technology to realize the integration of medical care; $\mathrm{Xu}$ Jiyong built a health management system for the elderly. Saad et al. designed a smart mattress and health all-in-one machine for home use, which can collect the physiological data of the elderly in real time and present these data in the client software, improving the quality and efficiency of 
elderly care services [13]. Roy et al. combined the characteristics of current senior care systems in the market and designed and implemented an integrated senior care management system, which added care functions, ordering functions, and self-service inquiries to the traditional focus on fee management model. R. B. Roy designed a new type of elderly care system, which uses sensors to collect the physical signs data of the elderly and, after processing and converting by the controller, transmits to the monitoring center through ZigBee network to complete the monitoring of the health data of the elderly at home [14]. In addition to intelligent products and services, the most important thing for smart elderly care is to build an integrated smart elderly care service platform centered on a cloud platform to achieve effective integration of elderly care resources and a high degree of matching services. Only based on building a mature and complete platform can Internet technology be used to provide intelligent elderly care services for the elderly.

To sum up, when designing elderly-oriented products for the elderly, we should not only consider the health data of the elderly but also combine the community, business, medical institutions, and government according to the actual needs of the elderly and extract the implicit information of the elderly from the massive data to provide personalized services for the elderly, to realize the real sense of intelligent elderly care.

\section{Digital Twin-Based Design of Complex Systems for Smart Aging}

3.1. Digital Twin System Design. In general, there is an inherent meaning to the data expressed in the information model, a practical application of the data. From the point of view of how the data is used, two forms are presented: the user view and the data manager view. The "user view" is the form of data defined from the perspective of the user currently using the data, and the "data manager view" is the form of data processed in the capacity of a data manager [15]. The abstracted view is the information model, which is somewhere between the user and the administrator. Therefore, the information model needs to be understood not only by users but also by data administrators. In the research process of this paper, the data is modeled for information integration, and a standard form is established to decide the necessary data that should be collected in the data collection system and the framework format of various data is specified.

To achieve rapid data transmission and reduce the resource consumption of computer equipment, under the condition that the information is not damaged and the usage function is not affected, the process of streamlining the $3 \mathrm{D}$ model in terms of geometry, bearing information, logic, and so forth is achieved by using the model facet reduction technology, information clouding technology, and so forth. This may be related to the poor use of computers and smartphones by the elderly. In the actual research process of the author, I found that some elderly people do not know much through websites and WeChat channels, but they know a lot through TV and friends around them, as well as through other channels such as newspapers and radio. This is also for future publicity work. The development provides a good idea to continue to maintain a better understanding of community publicity methods and at the same time to further increase the intensity of other publicity channels. These means are called lightweight processing. After the 3D modeling software draws the part diagram, the created part model generally has problems such as an excessive number of geometric vertices and many face slices, which has a great impact on the computer performance and memory and has a great test on the real-time simulation modeling, while reducing the user experience [16]. In the simulation modeling of this paper, the drawn part model needs to be lightened to reduce the time of loading the geometric model and the time of changing the simulation model on the simulation platform and to improve the overall display of the system. $3 \mathrm{~d}$ sMax software is chosen here. In the digital twin, the data drive realizes the real-time dynamic consistency between the physical object and the virtual model, and, through the OPC UA data collection of the industrial robot, the data is transmitted to drive the respective corresponding models in the virtual space, so that each part model in the virtual space maintains the same real-time state with the industrial robot or relies on the historical collection data to reproduce the operation condition of the industrial robot in a certain time state. The operation mechanism of the system is shown in Figure 1.

The digital twin model should be built by defining a unified data structure. First, based on the import of solid CAD models, the appearance, geometry, motion structure, and joint hierarchy of the model are defined. Then, in the face of different device types and diverse functions, the corresponding virtual service functions should be realized through the secondary development of scripts, such as the positive and negative solution of robot motion, the physical interaction between various tool heads and workpieces, the posture positioning of AGVs, and the morphological changes and information description of products. In this way, a generalized object-oriented modeling approach is refined for key industrial equipment to facilitate rapid project development and the establishment of a massive model library. The embedded intelligent elderly care service emphasizes the choice of a service method, based on the Internet of Things, big data, and cloud computing, the network is the link, the smart product is the terminal, and the integrated technology is used to match the service with the demand, which not only alleviates the problem of insufficient human resources but also provides high-quality elderly care services more conveniently and efficiently. In addition to this, the models are to establish data-driven interaction interfaces, thereby enabling the mapping of entities in digital space. The functional construction of twin models in the digital space is the engine for the implementation of digital twin systems. As an important concept of the Industrial Internet, the fundamental problem of the digital twin is to solve the problems of access to multiple types of devices from various manufacturers, the resolution and format unification of multiple data types, massive data 


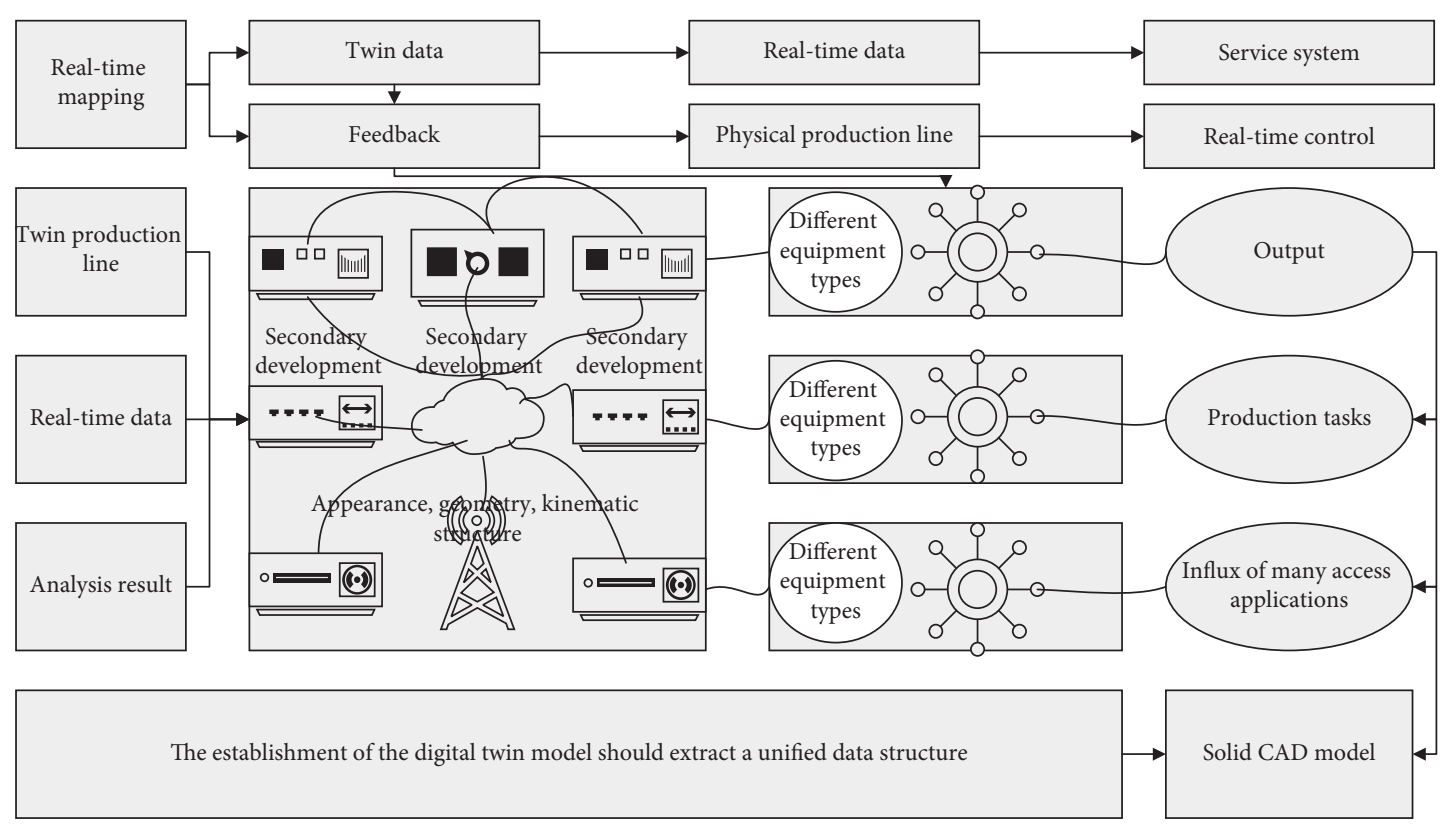

FIGURE 1: Schematic diagram of the operation mechanism of the digital twin system.

management, and analysis and computation. To ensure realtime interaction between the twin model and the field, a stable, rapid, and secure communication method is particularly important. For large scenarios with diverse devices, where there is a large amount of parallel and heterogeneous data, several common multisource data type parsing technologies for Industrial Internet, such as OPC UA, Modbus, and CAN bus, can effectively establish upstream and downstream communication. The communication control center should choose suitable communication protocols and common data exchange formats such as XML or JSON according to the communication situation of field devices to establish a bridge between upstream real-time information and downstream feedback data in the field. It can be said that multisource heterogeneous data processing technology is the basis for the realization of a digital twin system.

To comprehensively assess the quality of digital twin models, this chapter is designed to use the four following evaluation criteria for a comprehensive multidimensional assessment, which are Model Accuracy Ratio (MAR), Root Mean Square Error (RMSE), Variance Interpretation Rate (VIR), and Pearson's Correlation Coefficient (PCC). Refer to the formula in the introduction to the basic concepts of the digital twin model. The stand is the quantity, for example, the yield of the specified product, and $x$ is the prediction label (the independent variable). $y$ is the true value of the validation data set, $y$ is the mean, and MAR is the predicted value calculated by the training model. Big data of life services can provide intelligent life care support for the elderly, big data of cities, geography, and transportation can provide the elderly with intelligent travel, tourism, and transportation support, and big data of online media can provide intelligent support for the elderly: cultural, entertainment, and pension financial support. In summary, all levels of elderly care services are inseparable from the collection and analysis functions of big data, so big data is the core technology of smart elderly care.

$$
\text { MAR }=1+\sum_{i=1}\left|\frac{y_{i}-1}{y_{i}}\right| \text {. }
$$

During the training of a digital twin model using the LightGBM algorithm, the algorithm automatically calculates an important metric that reflects the importance of each data dimension leading to an increase in the total information gain of the model and corresponds to each data dimension in the form of an index after the model is finally formed. In the case of using a regression model, the information gain per bifurcation corresponds to the value of the reduction in the loss of a particular performance metric, such as the reduction in mean squared error. If the LightGBM algorithm uses a regression model in the construction of the model, where the $X$ feature is called $n$ times and each time the first order gain is $g$, the importance index $I$ of the feature can be represented by the following equation:

$$
I_{\alpha}=\sum_{i=1} \ln _{g^{i}}
$$

According to the complexity of the production system twin model, a connection and verification scheme is proposed. Firstly, it is necessary to obtain the equipment parameters, process parameters, layout parameters of the production line, and so forth as the basis for model construction and simulation. Based on the completion of the construction of the equipment monomer function, the input/output accuracy, confidence, sensitivity and precision, and motion characteristics (speed, spatial position, etc.) of the model deduction process are verified and corrected, followed by the organic connection of the equipment according to the process flow in the previous section. Based 
on the completion of the single function of the equipment, the input/output accuracy, confidence, sensitivity and precision, and motion characteristics (speed, spatial position, etc.) of the model in the model deduction process are verified and corrected, and then follow the previous section. The process flow connects the equipment organically. Various signals defined in the model can be connected freely, which is like communication in physical space and is more flexible. In the production process, the flow of parts and products is the main line of operation and relies mainly on a uniform organization pallet. By setting up a series of work processes at stations along the pallet flow path, the corresponding signals of the work process are triggered at each station as the pallets flow into it, thus controlling the corresponding equipment to perform different operations. In a specific case, the single device triggers the corresponding action by defining different trigger signals to achieve different responses to the incoming material. The individual devices in the cell are interconnected at the signal logic level according to the process flow and verified and corrected with the working time of each station as shown in Figure 2, which is mainly a comprehensive comparison and correction of the motion performance of the conveyor speed, the processing speed of the device, and the movement speed of the robot. In the connection and verification of the upper cell block to the system level, scripts are used to simulate the production signals to control their inputs, and the logic and rules are verified and corrected by comparing the statistical data with the actual data. After the logical connection and verification from the single device modeling to the whole production line, the overall twin model of the production line is finally constructed, which shows some of the connection signals between devices, and this model has the function of a real production line. As an important concept of the Industrial Internet, the basic problem of digital twins is to solve the problems of the access of multiple types of equipment of various manufacturers, the analysis of multiple data types and the unification of formats, the management of massive data, and the analysis and calculation of them. To ensure the real-time interaction between the twin model and the site, a stable, rapid, and safe communication method is particularly important.

3.2. Complex Model Construction for Smart Aging. The core concept of smart elderly care is "people-centered," and all work should start and end with meeting the needs of the elderly, relying on the Internet, big data, cloud platforms, and other information technology, combined with the needs of the elderly, through the combination with the traditional elderly care model, to provide the elderly with a full range of elderly care services. Smart senior care under big data technology has a precise service delivery strategy. It has a great impact on the performance and memory of the computer and has a great test of the real-time nature of simulation modeling and at the same time reduces the user experience. In the simulation modeling of this article, the drawn part model needs to be light-weighted to reduce the time of geometric model loading and the time of simulation

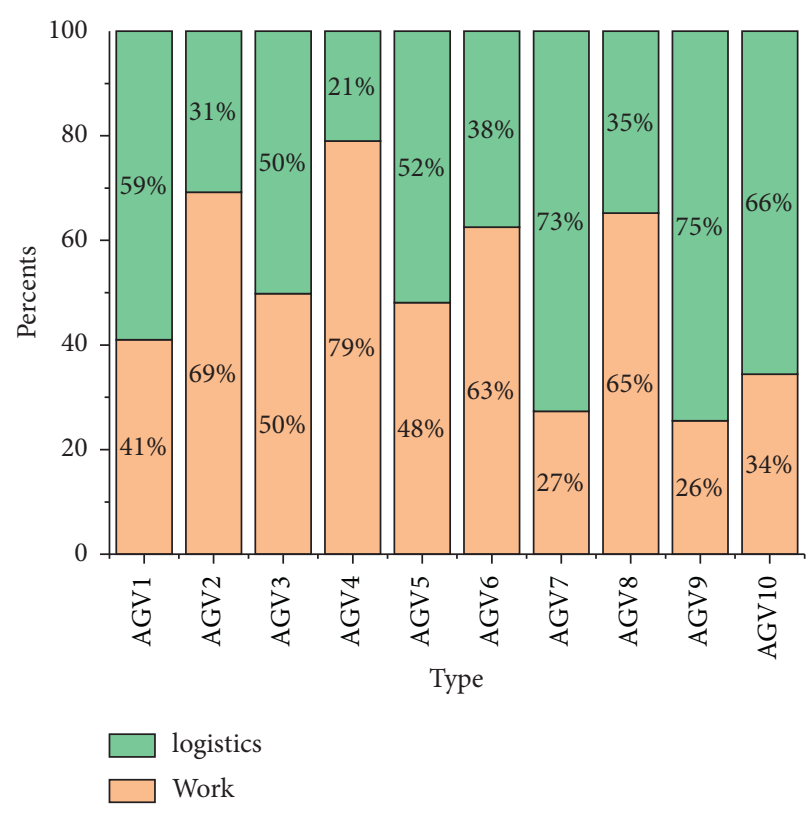

FIgURE 2: Work hours at each station of the digital twin system.

model change on the simulation platform and improve the overall display effect of the system. Here, $3 \mathrm{~d}$ sMax software is used. For example, big data of life services can provide wise life care support for the elderly, big data of city, geography, and traffic can provide wise travel, tourism, and traffic support for the elderly, and big data of network media can provide wise cultural, entertainment, and financial support for the elderly [17]. In summary, all levels of senior care services cannot be separated from the collection and analysis function of big data, so big data is the core technology of smart senior care. Smart senior care has strong inclusiveness; it takes service as the core and integrates various industries such as the Internet, service industry, real estate, and finance, thus forming a senior care service industry chain. This is the inevitable development trend of the senior care industry in the new era. The smart senior care service system includes three segments: intelligent devices, online service platform, and offline service circle. After the elderly wear the smart device, the smart device collects information and transmits the elderly's life data, health data, exercise data, consumer demand, and so forth to the online service platform; in short, the elderly and their families form a closed loop with the smart device, the online service platform, and the offline service circle, whose operation path is shown in Figure 3.

Relying on the Internet and big data platforms, smart senior care serves the elderly by integrating and consolidating useful information networks and using relevant smart technologies. Specifically, a smart senior care service platform is established to archive the basic information and service needs of the elderly; collect and integrate other senior care service information resources in the surrounding area, and interconnect the smart senior care service platforms among communities; provide life management and services for the elderly according to their needs; and meet their "socialized" needs. Embedded in the smart senior care service, more emphasis is placed on a service choice, based 


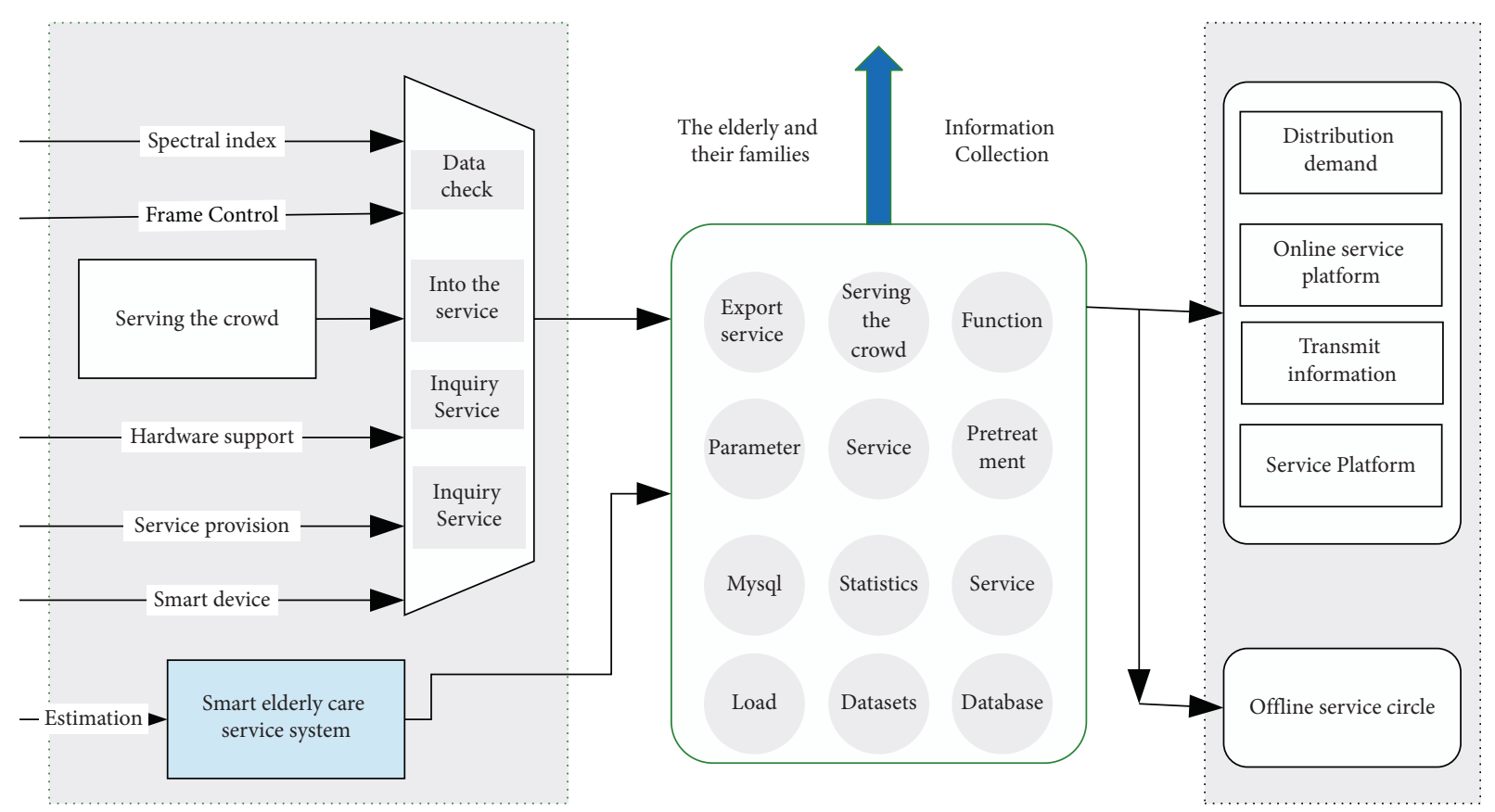

FIgURE 3: Closed-loop diagram of the smart aging system.

on the Internet of Things, big data, cloud computing, network as a link, smart products as the terminal, using integrated technology, the service and demand correspondence, not only to alleviate the problem of human resources shortage but also more convenient and efficient to provide high-quality senior care services. Smart senior care can be used not only in community-based home care services but also in institutional care, but the key that distinguishes it from other senior care services is that smart senior care takes advantage of information technology and applies integration technology to the process of senior care services. Therefore, the future of senior care should be replaced by intelligent senior care, which integrates public resources and social service resources by establishing a smart senior care service platform to provide diverse and personalized senior care services including daily life care, safety, and medical care, as well as recreational support for the elderly. Information technology-mediated intelligent elderly care will greatly improve the quality of elderly care services, ease the burden of staff, and solve many of the problems faced by community-based and institutional elderly care. To realize the rapid transmission of data and reduce the resource consumption of computer equipment, under the condition that the information is not damaged and the use function is not affected, the model patch reduction technology, the information cloud technology, and other means are used to realize the three-dimensional model in the geometry and carrying information. The simplification of the above processes and logic is called lightweight processing.

$$
\mathrm{PCD}=\frac{\mathrm{DW} / \mathrm{WD}}{M_{0}} .
$$

We have also conducted a survey on the ways of knowing about smart aging among the survey respondents, and, among the six choices of ways of knowing listed, each survey respondent can choose one or more options according to their actual situation, and the final result can be found that more of them are known through community publicity channels, and the websites and WeChat are not as good as the publicity methods, and this may be related to the fact that the elderly are not good at using computers and smartphones. In the actual research process, I found that some older people do not know much through the website and WeChat but through television and friends, as well as through other channels such as newspapers and radio. "User view" is a form of data defined from the perspective of the user who currently uses the data, and "data manager view" is a form of processing data with the identity of a data manager. The abstract perspective is the information model, which lies between the user and the administrator. This also provides a good idea for future publicity work; you can continue to maintain a better understanding of the community publicity methods and at the same time the strength of other publicity channels can further be increased (Figure 4).

Nonfunctional requirements are the characteristics that a software product must have to meet the business needs of users and are in addition to functional requirements. The definition of a nonfunctional software product not only determines the quality of the product but also influences the definition of functional requirements of the product to a large extent. Nonfunctional requirements analysis focuses on the following:

(1) Functionality, which refers to a set of attributes that satisfy formulated requirements and their nature, which include explicit or implicit requirements. Specifically, these include suitability, accuracy, interoperability, compliance, and security. 


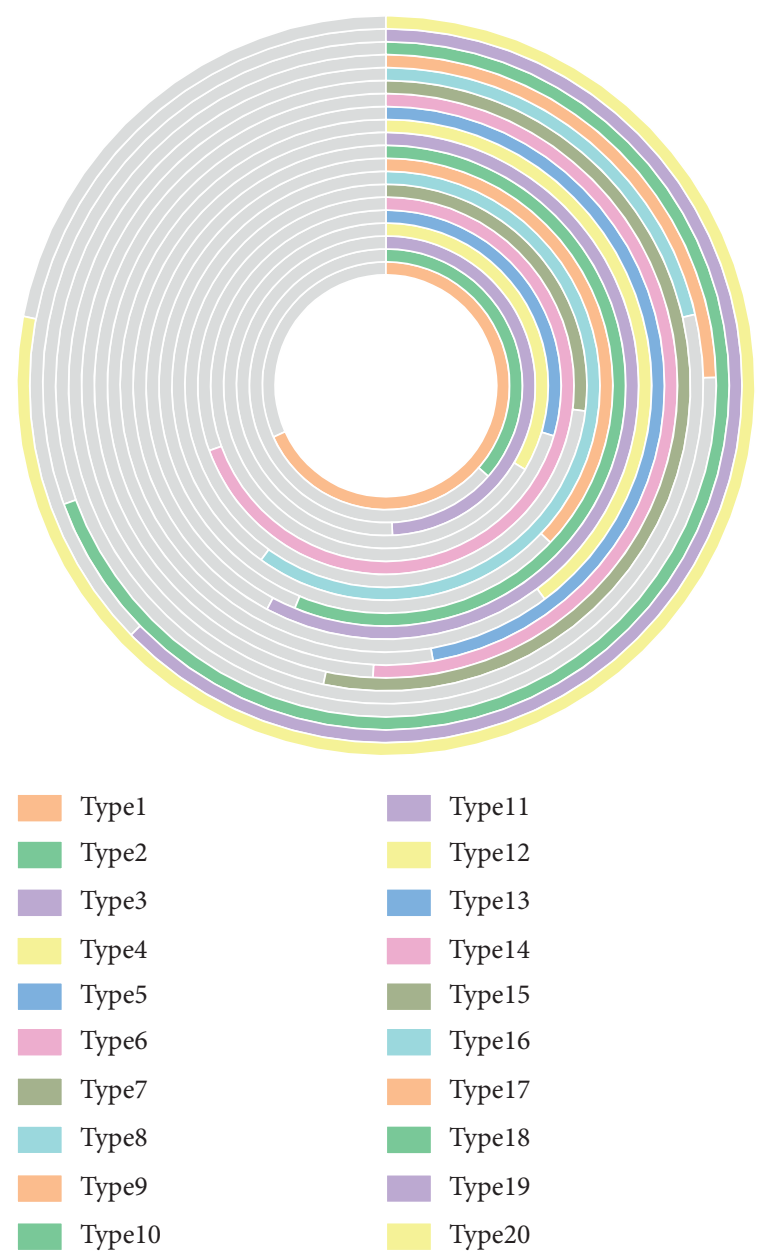

FIgURE 4: Statistical map of the pathways to understanding smart aging.

(2) Reliability, a set of attributes that refer to the ability of software to maintain its performance level over a specified time and under specified conditions. Specifically, they include maturity, fault tolerance, and ease of recovery.

(3) Ease of use, which refers to the user's effort to master the basic operation of the software and the evaluation of the ease of operation of the software attributes. It includes the following aspects: easy to understand, easy to learn, and easy to operate.

(4) Efficiency, a set of properties related to the resources used for the performance level of a system under specified conditions. It includes both time and resource characteristics.

(5) Maintainability, a set of attributes that refer to the time required for a system to make specified functional changes. These include ease of analysis, ease of change, stability, and ease of testing.

This part is usually the easiest area for the development team to invest time and cost in, such as dynamic properties to support web interface generation. Such requirements involve technical functional requirements to improve the maintainability of the system.

$$
\mathrm{EP}=\{\operatorname{Empty}(r)-\varphi\} .
$$

In addition to intelligent products and services, the most important thing is to build an integrated intelligent senior care service platform centered on a cloud platform to achieve effective integration of senior care resources and high matching of services. Only based on building a mature and perfect platform can we use Internet technology to provide intelligent senior care services for the elderly. The smart community management platform applied to the elderly is specifically divided into an elderly care service platform, a volunteer platform, and a social platform for the elderly. The elderly care service platform includes an intelligent medical care terminal, wireless care network, and backstage monitoring platform to provide elderly life care service and medical care integration service [18]. The Etchemendy research group pays more attention to the emotional changes of the elderly while paying attention to the health data of the elderly. When the elderly is in a depressed or negative emotional state, the system can accurately sense the emotional difficulties of the elderly and evaluate the mental state of the elderly to provide the most suitable treatment for the elderly. First, in the medical care terminal, intelligent devices, such as fall detection carpet, sleep monitor, mattress sensor, intelligent robot, and health hut, are used to collect real-time health and safety data of the elderly; then, the data is transmitted to the background monitoring platform through wireless communication networks such as routers and WIFI; the background focuses on the dynamics of the elderly through real-time monitoring and provides a realtime alarm in case of danger; finally, the collected basic information of the elderly combined with the demand is sent to the background monitoring platform. Finally, the basic information collected from the elderly combined with the demand will be sent to the intelligent community management platform for the service application and acceptance of related services. The integrated intelligent elderly service platform with technology as the means and wisdom as the medium can not only facilitate the management of staff and improve the efficiency of workers but also pay attention to the physiological and psychological needs of the elderly in all aspects and provide them with high-quality elderly services in a continuous manner.

$$
\lambda=0.1\left(\frac{d}{K_{0}}\right) 2 .
$$

\section{Analysis of Results}

4.1. Digital Twin System Results. Model predictive control (MPC) algorithm is an advanced control technique based on display predictive models that use model multistep prediction, rolling optimization, and feedback correction strategies to predict and optimize the future dynamic response output of an object. The predictive model is an 
important part of predictive control, and its main function is to predict the future state or output of the controlled object based on its historical information and future inputs, especially for nonlinear predictive control systems. Predictive control has no strict mandatory requirements on the structural form of the predictive model, but its control effect largely depends on the performance of the predictive model. Obtaining a predictive model that accurately reflects the future changes in the dynamic characteristics of the system is a prerequisite for achieving optimal control. Predictive control is an optimization-based control algorithm that uses a certain performance index to evaluate the control effect of the controlled object. The optimization in predictive control is a kind of rolling optimization with a finite time, and the optimization process is not performed offline once but repeatedly online. The test is a performance test, using the testing tool to complete the recording of the performance data during the operation of the system, with the values of $\mathrm{CPU}$ usage, memory usage, and other parameters recorded during the experiment as the benchmark for assessment, and the performance test time is one hour. The evaluation criteria are as follows: CPU usage needs to be less than $40 \%$ of the actual CPU total, and memory usage needs to be less than $30 \%$ of the actual memory total. In this experiment, the system is monitored using the resource monitor in the task manager, and the overall performance test results of the system are monitored and recorded for the CPU usage data of key nodes in the performance test process. The system test performance data results are shown in Figure 5 to meet the standard CPU usage needs to be less than $40 \%$ of the actual total CPU.

The digital twin has initially entered the stage of practice and application from the conceptual model stage, and the description and research of its nature and connotation have also been more in-depth, highlighting the hierarchical, integrated, superrealistic, extensive, consistent, and accessible nature of the digital twin. In the digital space, each part and level require different statistics and different statistical methods, but, in general, a bottom-up approach is needed, with the upper level using the statistics of the lower level for deeper calculations. For the equipment, its joint values, spatial coordinate values, cylinder values, and other motion data are already stored through the data service program and are not processed here in the simulation space. The key statistics of the equipment are to be realized by its state information; each state is to be given according to the realtime data of the equipment working in the field, and the digital space counts the changes of the working state of the equipment according to the correspondence between the state changes of the equipment and the time flow, from which the utilization rate of the equipment and so forth can be further calculated. The upper layer can further analyze the statistical data of all the equipment, service components, and products in the production line and get the data such as comprehensive utilization rate of the equipment, product qualification rate, and planning status of the whole production line, which can provide support for further use of algorithms to achieve optimal control of the production line [19]. The key statistical data in the

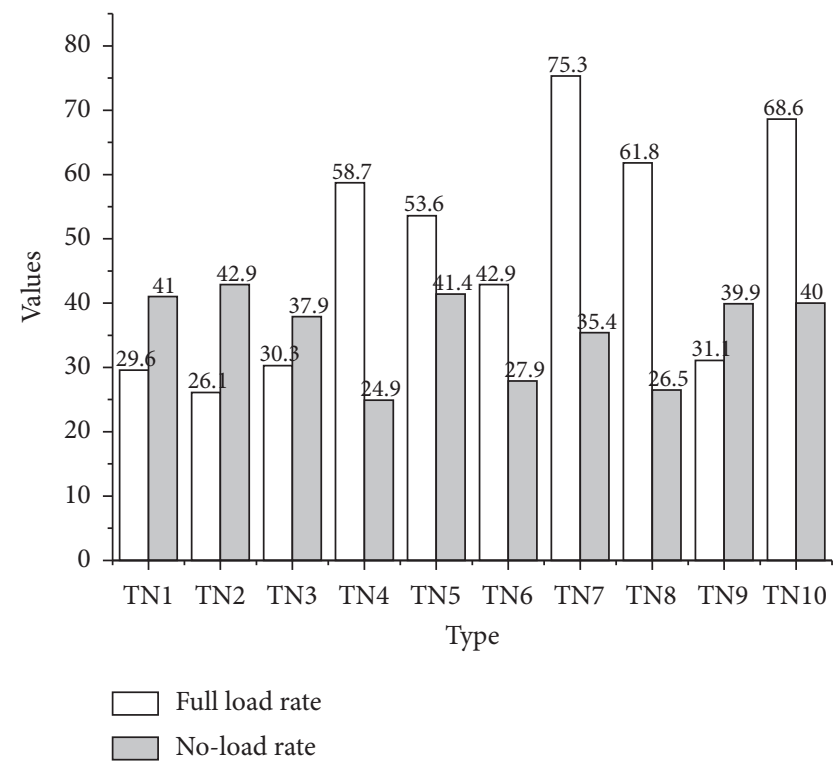

Figure 5: Performance test data graph.

production line are displayed, and, in addition to the storage of the above statistics, the software provides line graphs, pie charts, Bar charts, and other basic visualization charts, robot joint values, state equipment ratios, part counts, and so forth can be visualized, as shown in the display in Figure 6 for the statistical data line chart. In addition to visualization in the form of charts, the screen model in the digital space uses functional scripts to implement a real-time display of statistical data, showing key data for each workstation.

The data generated during the operation of the digital space is constantly updated with the flow of time and is initialized when the system is restarted or ended. To make subsequent use of the twin data in the digital space mentioned above, the storage of the data is also required. The data storage in the digital space takes two forms: one is the output of Excel reports; the other is the storage of the twin data in a database. The real-time drive data in the digital space has been stored through the data service program, and no further storage operations will be performed here, mainly for the data obtained from calculations and statistics during the operation of the system. The twin data generated in the digital space can likewise be divided into three types, corresponding to different storage methods for product data, equipment data, and production system data. This article combines the content and characteristics of elderly care services and innovates the methods and contents of elderly care services. That is, taking the community as the carrier, while embedding functions, resources, and contacts, while embedding intelligent technology based on the Internet and the Internet of Things, it can meet the physical and psychological needs of the elderly, deal with social risks, and improve the quality of elderly care services.

$$
\mathrm{HV}=\mathrm{PE}=\frac{1.5844}{d^{2}} .
$$




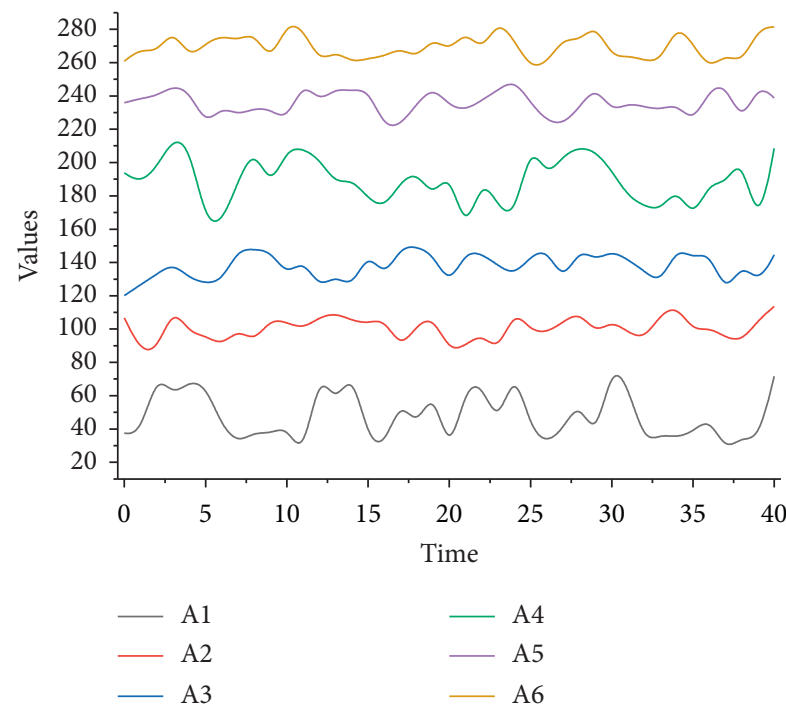

FIGURE 6: Schematic diagram of the statistical folding line.

The output of the data transfer mainly consists of the RESTful server thread and the request processing thread, which provides the server opening function and enables the request listening. The request processing thread is required to classify and process the requests sent by the virtual workshop and other service systems and return the processing results to ensure the accuracy and integrity of the returned data. This import method can effectively avoid the loss of model information during the import process, perfectly realize the data conversion, ensure the quality and geometric information of the components, facilitate the editing after import, and import fast and efficiently. Its conversion method is as follows:

(1) Save the assembled model in SOLIDWORKS in a .parasolid format

(2) Open ADAMS and open the SOLIDWORKS saved .parasolid format file

(3) Define the model's name and import the model

After a comparative analysis of the spectrograms obtained in the postprocessing module, the results of the simplified model simulation are consistent with the simulation results of the structure containing the box and so on.

The data output part is mainly designed with RESTful architecture, by opening the RESTful server, listening to the data requests from other systems, and when receiving data requests from other systems, querying the relevant data in the object database according to the specific request content, and timely outputting the data in XML/JSON format to the requesting system to complete the data output.

4.2. Smart Aging Model Implementation. The physiological needs of the elderly group include clothing, food, housing, transportation, and daily care. For the needs in this area, the module of the old shopping mall of daily necessities designed for the elderly and the module of domestic services designed for the elderly who have lost the ability to take care of themselves or have no one to take care of them can be built within the system. In terms of security needs, the system can be built with electronic health information files and online consultation modules for the elderly to meet their health needs promptly [20]. The purpose of this platform is to connect individuals, communities, corporate service providers, and medical institutions through the comprehensive service platform of "elderly" and "health" to meet the diversified and multilevel needs of the elderly. Older users can access relevant information through the front section of the Internet and provide expert consultation services for solving various problems they encounter. Medical experts can provide answers to the elderly's consultation questions through the expert oral consultation system. Enterprises can release relevant product information through the senior citizen mall. Therefore, the functional requirements of the platform mainly include the functional requirements of four parts: the elderly, medical experts, enterprises, and administrators.

Technological innovation can affect organizational costs and thus the outcome of collective action. Digital technologies offer a way to reduce the constraints of time and space, and their impact on the cost of delivering products and services will lead to significant changes in the mix of actions that any collective can achieve. Collective action is increasingly dependent on the encoding, communication, and exchange of information, and networks have reduced the distances of space and time, with communication and interaction across time, place, and domain becoming the norm. According to the six degrees of separation theory, any stranger can be met by up to six people, creating a social environment in which communication is easy. It is to make full use of physical models, sensors, operating history, and other data, integrate multidiscipline, multiphysical quantities, and multiscale simulation process, realize the mapping of physical space in digital space, and reflect the whole life of corresponding physical equipment and products. The use of digital technology contributes to the formation of "inclusive" collectives that advance the public service goals supported by the "more is better" rule. This means that, instead of higher decision-making costs and higher potential deprivation costs, the increase in collective membership benefits from increased membership, and the cost of collective action decreases as the goals of interest are shared (as shown in Figure 7). As organizational costs fall, the size of the group achieving collective action becomes larger and the likelihood of collective action elevates.

"Collective" refers not only to the number of people who express their views but also to the consensus achieved among the different points of view of interest. Collective action is a negotiation process and its success depends on the quality of dialogue, interaction, and effective negotiation. Citizens are the most important monitoring subjects in the public sphere, and, by expressing their individual opinions and generating social discussion in the network, they can be regarded as "negotiators of public opinion," which can stimulate the need for collective action and rationalize public decisions through continuous discursive negotiations in the public sphere. Traditional elderly care service models mainly 


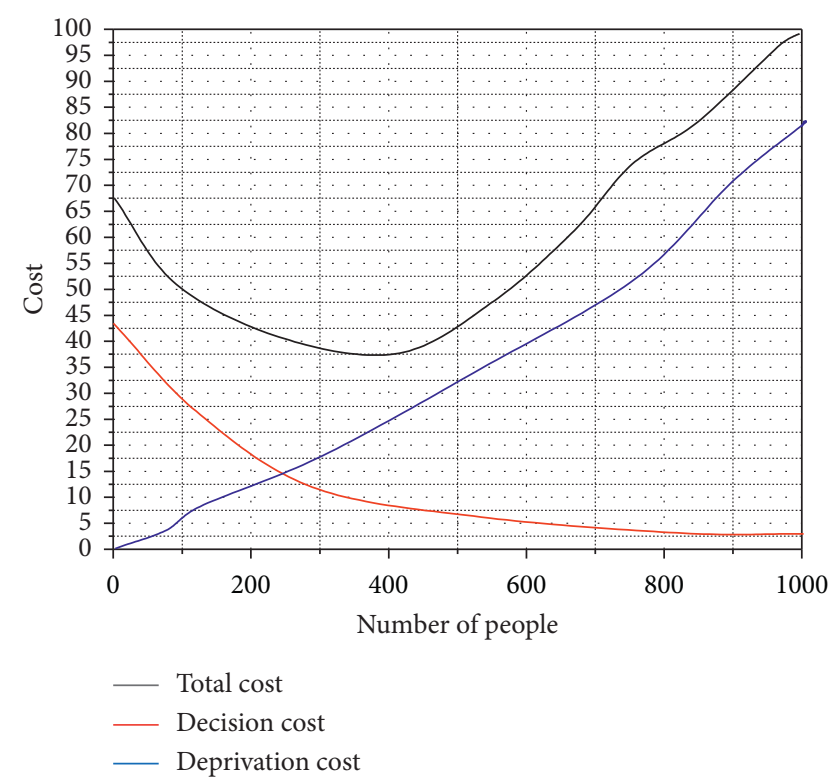

Figure 7: Schematic representation of the cost of inclusive, practical group action.

include family elderly care, community elderly care, and institutional elderly care. With the "4-2-2" family structure and large-scale aging, it is difficult for the traditional old-age care model to adapt to the needs of the old-age care in the new era. Promoting the development of the elderly care industry, stimulating consumption, and deepening the supply-side structural reform "two legs" must work at the same time. The networked transmission of individual opinions into social consciousness will likely trigger timely improvements in public services.

A cross-sectional analysis of the age of older people and the services they need (Figure 8) shows that older people in different age groups have different needs for elderly services. In terms of medical care, all age groups have a high demand for medical care; in terms of life care, the demand of those aged below 60 years and those aged $60-70$ years is $28 \%$, but the demands of those aged $70-80$ years and $80-90$ years reach $55 \%$ and $89 \%$, respectively. In terms of spiritual comfort, the demand of the elderly below 60 years of age is about $40 \%$, and the demand of the elderly aged $60-70$ years is $70 \%$, which means that the demand of the elderly for spiritual comfort has reached the peak, while the demand of the elderly aged above 70 years is about $55 \%$. Health is more important to them. In terms of end-of-life care, the demand is higher among those aged $80-90$ years, accounting for $39 \%$, while those below 80 years of age have no demand for the time being; in terms of financial care for the elderly, the demand is highest among those below 60 years of age, at $20 \%$, and among those aged $60-70$ years, at $11 \%$, while those aged above 70 years have almost no demand. In conclusion, the different levels of needs of the elderly are related to the age of the elderly; the older the elderly group are, the more importance they attach to instinctive needs, and the younger elderly group are, the more importance they attach to spiritual needs. Embedding smart elderly care supported by

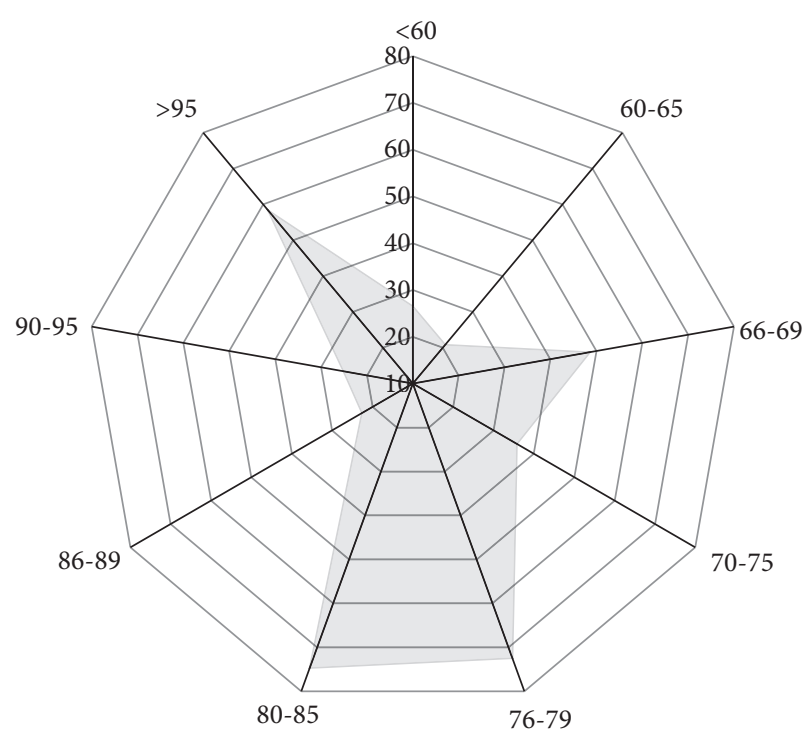

FIGURE 8: Results of the cross-tabulation of age and aged care needs services for older people.

informatization and intelligence into the elderly care service system is not only to actively respond to the objective needs of population aging but also to cater to the development trend of the information society and seize the opportunities of the times to promote the level of social governance.

The conceptual structure design phase is the key to the overall database design. By classifying, aggregating, and generalizing the type, scope, and quantity of data used in the system and their exchange in business activities, an abstract conceptual data model is created to describe the information structure of the system, the flow of information, the interconstraint relationships between information, and the requirements of each ministry for information storage, query, and processing. The model built should avoid the concrete implementation details of the database on the computer and represent it in an abstract form. In this paper, the E-R diagram approach is used to describe the conceptual model of the system. In this information service platform, the entities are mainly in several categories such as elderly people, health records, experts, companies, commodities, orders, system administrators, and life and entertainment information.

\section{Conclusion}

This paper firstly introduced the current situation of the elderly problem, put forward the current elderly model of security care, health management, life care, leisure and entertainment, affectionate care, and other aspects of service problems, combined with the current exploration of the intelligent elderly model and the promotion of information technology on medical elderly services, and proposed the construction of a complex model of intelligent elderly based on digital twin; digital twin design is completed using Web for object-oriented analysis modeling. Determine the requirements of the platformthrough demand analysis and establish the example diagram for system participants and 
drawing according to the requirements.Establish the class diagram of the static structure model and the activity diagram of the dynamic structure model of the system, analyzethe behavior of the system, and clarify the workflow of the system. The next king of work is the design and implementation of the Web pages. First, the web architecture and system functionality of the system is given in conjunction with the digital twin system architecture. Second, the relational database suitable for this system is designed with the system architecture and functionality. Finally, the interface implementation of the system is given. In the process of system design and development, along with thorough preparation and a lot of effort, but due to the limited ability of their own technical and theoretical aspects, the system still has problems that need to be improved. The mature industrialization of the industry related to smart aging cannot be separated from the government supervision of enterprises; this system should also strengthen the cooperation with the government in this regard.

\section{Data Availability}

The data used to support the findings of this study are available from the corresponding author upon request.

\section{Conflicts of Interest}

The author declares that there are no known competing financial interests or personal relationships that could have appeared to influence the work reported in this paper.

\section{References}

[1] Z. Cunbo, J. Liu, and H. Xiong, "Digital twin-based smart production management and control framework for the complex product assembly shop-floor," International Journal of Advanced Manufacturing Technology, vol. 96, no. 1-4, pp. 1149-1163, 2018.

[2] S. Meraghni, L. S. Terrissa, M. Yue, J. Ma, S. Jemei, and N. Zerhouni, "A data-driven digital-twin prognostics method for proton exchange membrane fuel cell remaining useful life prediction," International Journal of Hydrogen Energy, vol. 46, no. 2, pp. 2555-2564, 2021.

[3] P. K. Kwok, M. Yan, T. Qu, and H. Lau, "User acceptance of virtual reality technology for practicing digital twin-based crisis management," International Journal of Computer Integrated Manufacturing, vol. 34, no. 7-8, pp. 874-887, 2021.

[4] Q. Wang, W. Jiao, P. Wang, and Y. Zhang, "Digital twin for human-robot interactive welding and welder behavior analysis," IEEE/CAA Journal of Automatica Sinica, vol. 8, no. 2, pp. 334-343, 2020.

[5] E. Yildiz, C. Møller, and A. Bilberg, "Demonstration and evaluation of a digital twin-based virtual factory," International Journal of Advanced Manufacturing Technology, vol. 114, no. 1, pp. 185-203, 2021.

[6] R. Minerva, G. M. Lee, and N. Crespi, "Digital twin in the IoT context: a survey on technical features, scenarios, and architectural models," Proceedings of the IEEE, vol. 108, no. 10, pp. 1785-1824, 2020.

[7] L. Lei, G. Shen, L. Zhang, and Z. Li, "Toward intelligent cooperation of UAV swarms: when machine learning meets digital twin," IEEE Network, vol. 35, no. 1, pp. 386-392, 2020.
[8] B. Zavareh, H. Foroozan, M. Gheisarnejad, and M. -H. Khooban, "New trends on digital twin-based blockchain technology in zero-emission ship applications," Naval Engineers Journal, vol. 133, no. 3, pp. 115-135, 2021.

[9] P. Jain, J. Poon, J. P. Singh, C. Spanos, S. R. Sanders, and S. K. Panda, "A digital twin approach for fault diagnosis in distributed photovoltaic systems," IEEE Transactions on Power Electronics, vol. 35, no. 1, pp. 940-956, 2019.

[10] Y. Fang, C. Peng, P. Lou, Z. Zhou, J. Hu, and J. Yan, "Digitaltwin-based job shop scheduling toward smart manufacturing," IEEE Transactions on Industrial Informatics, vol. 15, no. 12, pp. 6425-6435, 2019.

[11] K. Wang, Q. Hu, M. Zhou, Z. Zun, and X. Qian, "Multi-aspect applications and development challenges of digital twindriven management in global smart ports," Case Studies on Transport Policy, vol. 9, no. 3, pp. 1298-1312, 2021.

[12] D. Chen, D. Wang, Y. Zhu, and Z. Han, "Digital twin for federated analytics using a bayesian approach," IEEE Internet of Things Journal, vol. 8, no. 22, pp. 16301-16312, 2021.

[13] A. Saad, S. Faddel, T. Youssef, and O. A. Mohammed, "On the implementation of IoT-based digital twin for networked microgrids resiliency against cyber attacks," IEEE Transactions on Smart Grid, vol. 11, no. 6, pp. 5138-5150, 2020.

[14] R. B. Roy, D. Mishra, S. K. Pal et al., "Digital twin: current scenario and a case study on a manufacturing process," International Journal of Advanced Manufacturing Technology, vol. 107, no. 9, pp. 3691-3714, 2020.

[15] X. Xiangdong, L. Bo, and G. Jiannan, "Asset management of oil and gas pipeline system Based on Digital Twin," IFACPapersOnLine, vol. 53, no. 5, pp. 715-719, 2020.

[16] B. Schleich, N. Anwer, L. Mathieu, and S. Wartzack, "Shaping the digital twin for design and production engineering," CIRP Annals, vol. 66, no. 1, pp. 141-144, 2017.

[17] F. Tao, Q. Qi, L. Wang, and A. Y. C. Nee, "Digital twins and cyber-physical systems toward smart manufacturing and industry 4.0: correlation and comparison," Engineering, vol. 5, no. 4, pp. 653-661, 2019.

[18] D. Y. Jeong, S. H. Kim, I. B. Lee, U. H. Yeo, J. G. Kim, and S. J. Park, "A study on the implementation of digital twin architecture and detailed technology for agriculture and livestock industry," Journal of Broadcast Engineering, vol. 26, no. 4, pp. 398-408, 2021.

[19] T. Fei, C. Jiangfeng, Q. Qinglin, Z. Meng, Z. He, and S. Fangyuan, "Digital twin-driven product design, manufacturing and service with big data," International Journal of Advanced Manufacturing Technology, vol. 94, no. 912, pp. 3563-3576, 2018.

[20] J. Kim, W. Choi, M. Song, and S. Lee, "Design and implementation of IoT platform-based digital twin prototype," Journal of Broadcast Engineering, vol. 26, no. 4, pp. 356-367, 2021. 